\title{
Non Invasive Estimation of Left Ventricular Normalized Torsion Angle in Healthy Persons by Echo Tracking Algorithm: Short Axis View
}

\author{
Zahra Arab Baferani ${ }^{1}$, Manijhe Mokhtari-Dizaji ${ }^{*}$, Farideh Roshanali ${ }^{2}$ \\ ${ }^{1}$ Department of Medical Physics, Tarbiat Modares University, Tehran, IR Iran \\ ${ }^{2}$ Department of Cardiology, Day Hospital, Tehran, IR Iran
}

A R T I C L E I N F O

Article type:

Original Article

Article history:

Received: 13 Apr 2011

Revised: 22 Apr 2011

Accepted:16 May 2011

Keywords:

Echocardiography

Heart Ventricles

Echo-Planar Imaging

\begin{abstract}
A B S T R A C T
Background: Left ventricular (LV) torsion angle is a key parameter of cardiac performance but is difficult to measure. The purpose of this study is to describe a noninvasive imaging method for the assessment of these complex cardiac motions.

objectives: In this study, left ventricular torsion angle and normalized torsion angle were estimated in the short axis view for healthy persons.

Patients and Methods: We acquired basal and apical short axis left ventricular LV images in the short axis view for 14 healthy men to estimate LV torsion angle by echo tracking under a block-matching (BM) algorithm. By extracting the instantaneous changes in total displacement vector in the short axis view throughout a cardiac cycle, we calculated the instantaneous rotation and torsion angles in the short axis view. To account for differences in heart size, normalized torsion was calculated based on the radius of both the apical and basal slices in the end diastolic frame.

Results: Apical and basal rotation was measured from short axis images by automatic frame-to-frame tracking of grayscale echo patterns. The vertical and horizontal displacements of the apical level were more than those of the basal level. All data are expressed as mean \pm standard deviation (SD). Data was tested for normal distribution and homogeneity of variance by the Kolmogorov-Smirnov test (K-S) and Levene's test respectively. The peak rotation angles of the basal and apical levels and LV torsion angle in the short axis view were $8.0 \pm 1.6^{\circ}, 9.5 \pm 1.8^{\circ}$, and $17.3 \pm 2.5^{\circ}$, respectively. The normalized torsion was calculated as $7.8 \pm 1.3^{\circ}$, based on the basal maximum radius, the apical maximum radius, and the distance between the apical and basal levels.

Conclusions: We conclude that the normalized torsion angle is an important biomechanical parameter, because it is independent of heart size and distorted geometries in pathological ventricles.

Copyright @ 2011 Kowsar Corp. All rights reserved.
\end{abstract}

Implication for health policy/practice/research/medical education:

The importance of measuring LV rotation lies in its potential for early detection of pathology and the ability of torsion techniques to provide regional information. In this study, a non invasive imaging method available for the assessment of these complex cardiac motions was described.

- Please cite this paper as:

Arab Baferani Z, Mokhtari-Dizaji M, Roshanali F. Non Invasive Estimation of Left Ventricular Normalized Torsion Angle in Healthy Persons by Echo Tracking Algorithm: Short Axis View. Int Cardiovasc Res J.2011.5(4):127-33. DOI: 10.5812/icrj.4481

\section{Background}

Through recent developments in echocardiography, we can quantify myocardial motion and deformation (1).

\footnotetext{
* Corresponding author: Manijhe Mokhtari-Dizaji, Department of Medical Physics, Tarbiat Modares University, Tehran, IR Iran. Tel: +98-2182883893, Fax:+98-2188006544, E-mail: mokhtarm@modares.ac.ir

DOI:10.5812/icrj.4481

Copyright @2011 Kowsar Corp. All rights reserved.
}

Heart torsion is one of the biomechanical parameters that are sensitive to changes in regional and global left ventricular function (2). Left ventricular (LV) torsion occurs because cardiac myocytes branch and interconnect with neighboring myocytes in axial tracts, which are longitudinal strands supported within a matrix of fibrous tissue. These grains spiral from the apex to the base about the LV in helical arrangements, resulting in 
a complicated, 3D twisting motion during systole and an unwinding motion during early diastole. This structural arrangement is believed to be both energetically optimal and important for equal redistribution of LV stresses and strains. In LV torsion, the motion of the apex and base is described conventionally from an apex-tobase view down the longitudinal axis of the left ventricle, with clockwise rotations measured in negative degrees and counterclockwise rotations measured in positive degrees. When isovolumic contraction (IVC) begins, the apex initially rotates clockwise as the subendocardial myofibers shorten, but then, apical rotation reverses and becomes counterclockwise for the duration of systole as forces that are generated by subepicardial myofibers predominate. The magnitude of basal rotation is significantly less than that of apical rotation. When IVC begins, the base undergoes a brief counterclockwise motion due to subendocardial fiber shortening, which is followed by a sustained clockwise rotation for the rest of systole as subepicardial myofibers predominate. LV torsion is the sum of the magnitude of apical and basal rotations during systole, expressed in degrees or radians (3-5).

Left ventricular torsion during systole stores potential energy, causes uniform distribution of stress, and equilibrates myocardial fiber shortening. Systolic twist keeps the high intraventricular systolic pressure to a minimum; therefore, myocardial energy consumption will be limited (6). Different methods, such as implanted radiopaque markers, biplane cine angiography, sonomicrometry, optical devices, magnetic resonance imaging, and echocardiography, have been used to measure left ventricular torsion in the short axis view $(2,7-9)$.

Motion profile can be achieved by tracking echo patterns, frame by frame, in the cardiac cycle. Speckle tracking imaging, which is based on a BM (block-matching) algorithm, is one of the approaches for estimating motion in cardiac muscles. In a block-matching algorithm, it is assumed that the pixels within the compact block have similar motions and that the motion vectors that are assigned to the compact block are the average of motion vectors of pixels within the block (10-13). The purpose of this study is to describe a noninvasive imaging method to assess these complex cardiac motions.

\section{Objectives}

In this study, left ventricular normalized torsion angle was estimated in the short axis view in healthy persons.

\section{Patients and Methods}

Torsion angle $(\gamma)$ is measured most easily as the maximum angle attained during systole between apical $(\alpha)$ and basal $(\beta)$ short axis slices rotating in opposite directions (see Figure 1):

$$
\gamma=\alpha-\beta(1)
$$

Measurement of left ventricular torsion in the short axis view by motion detection that is based on echo tracking by BM algorithm was performed. Motion vectors in the cardiac cycle in the septal region were extracted by running the BM algorithm on consecutive frames. In the short axis view, we could extract displacement in the $\mathrm{X}$ and $Y$ directions. After detecting the center of the basal and apical levels in the end diastolic frame and motion vectors in the $\mathrm{X}$ and $\mathrm{Y}$ directions in the cardiac cycle, rotation angles in the short axis view at the basal and apical levels were extracted.

The problem with this method arises from non-uniform slice selection. Therefore, it is critical to use the same basal and apical slices when measuring and comparing torsion in the same patient on two dates.

In this work, basal and apical levels were defined in the short axis view at the mitral valve and distal level of Lwithout visible papillary muscles, respectively. Given the heterogeneity in papillary muscle prominence and apical trabeculation in humans and distorted geometries in pathological ventricles, this may not always be possible. To solve this problem, torsion can be normalized by dividing it by the distance between the selected apical and basal slices in the diastolic frame (h), which is in degrees per centimeter. To account for differences in heart size, normalized torsion can also be adjusted to include the radius of both the apical $\left(\mathrm{R}_{\text {apex }}\right)$ and basal $\left(\mathrm{R}_{\text {base }}\right)$ slices in the end diastolic frame as apex rotation arc and base rotation arc:

$$
\gamma=\frac{\alpha \times R_{\text {apex }}-\beta \times R_{\text {base }}}{h}
$$

ie, $\gamma, \alpha$, and $\beta$ are the torsion, apical rotation, and basal rotation angles in degrees, respectively. In this study, we assessed left ventricular torsion in short and long axis planes of 14 healthy men aged $46 \pm 5$ years. They did not have any history of heart disease, such as myocardial infarction, ventricular arrhythmia, and heart failure, and they were selected based on the Framingham study (14, 15); thus, all healthy subjects had a normal physical examination, ECG, and echocardiography. Systolic and diastolic blood pressures were recorded in the left radial artery in the supine position using a semiautomatic device (Riester 0124, Germany) prior to the echocardiographic studies. This study was approved by the ethics committee of Tarbiat Modares University.

All echocardiography studies were performed with a Vivid7 digital ultrasound scanner (GE, Milwaukee, WI, USA), equipped with an M3S transthoracic sector transducer with harmonic capability (1.5-4.0 MHz). The images were acquired with the subjects at rest and lying in the lateral decubitus position. Two-dimensional ECG images were superimposed on the images, and end diastole was considered at the peak R-wave of the ECG. The left ventricular end diastolic/systolic volumes (VED/VES), the distance between the selected apical and basal slices in the diastolic frame $(h)$, and the radius of both the apical $\left(R_{\text {apex }}\right)$ and basal $\left(R_{\text {base }}\right)$ slices in the end diastolic frame were measured in the short and long axis views of LV, and left ventricular ejection fraction (LVEF) was calculated using Simpson's biplane method by measuring end diastolic and end 
systolic volumes in 2D images. 2D echocardiography was performed using the standard parasternal short axis view according to the guidelines of the American Society of Echocardiography (ASE) $(16,17)$.

For each subject, we scanned basal and apical levels in the short axis view using a high-frame-rate (60 frame/s) harmonic B-mode with transmitted and received frequency of $1.7 \mathrm{MHz}$ and $3.4 \mathrm{MHz}$, respectively. Then, echocardiographic images of the short axis view at the mitral valve and apex planes were scanned throughout the four cardiac cycles and stored digitally in cine loop format in the memory of the scanner and transferred to a PC for postprocessing.

In this study, echo tracking in the consecutive frames was estimated by BM algorithm $(17,18)$. In the BM algorithm, given a block of pixels or reference block in the current frame, matching comprises finding the block in the next frames that best matches the block in the reference frame. The displacement vector is estimated by matching the information content of a measurement window with that of a corresponding measurement window within a search area, placed in the previous frame. The data were then exported to MATLAB for image processing in MATLAB, version 7.0.1 (Math Software Co., Mathworks, USA). Pixel size in the digitized ultrasound image was $0.4 \mathrm{~mm}$. The algorithm mentioned above was run to measure the instantaneous changes in the septal myocardial wall at the basal and apical levels relative to the probe in the short axis view for 10 runs. A repeatable error of less than $10 \%$ was accepted.
The instantaneous myocardial wall changes were extracted by calculating the difference between instantaneous changes of the septal wall between end diastolic phase with end systolic phase with a temporal resolution of $17 \mathrm{~ms}$. Motion vector (Cartesian coordinates according to frame number) of the ROI in the horizontal and vertical directions was obtained. Total myocardial wall displacement was calculated based on displacements in the horizontal and vertical directions. According to the recorded electrocardiogram and motion vectorswhich were accessed through the BM algorithm, the end diastole frame was extracted. Then, the center of the left ventricular in the short axis view was determined. In this study, we assumed that the central displacement in the cardiac cycle between the consecutive frames was ignorable. All data were expressed as mean \pm SD (standard deviation ). After verifying the normality and homogeneity of the variables, the paired sample t-test was performed with a $95 \%$ confidence interval $(P<0.05)$. Comparison of differences in the values was performed by paired samples t-test. Results were considered significant when the probability value was $<0.05$. To evaluate the method, the percentage of the coefficient of variation was calculated.

\section{Results}

The demographic and echocardiographic measures of 14 subjects ( $46 \pm 5 \mathrm{yr}$ ) are given in Table 1 as mean \pm SD. The subjects were comparable with regard to age, heart rate, body mass index (BMI), systolic and diastolic blood

\begin{tabular}{ll}
\hline Table 1. Mean \pm SD of Demographic and Echocardiographic Measures of the Subjects & \\
\hline Variables & Amounts, mean \pm SD \\
\hline BMI: Body mass index, kg/m2 & $25 \pm 2$ \\
Heart rate, beats/min & $72 \pm 8$ \\
Diastolic blood pressure, mmHg & $80 \pm 10$ \\
Systolic blood pressure, mmHg & $113 \pm 10$ \\
Interventricular septal diastole thickness, mm & $11.9 \pm 0.8$ \\
Posterior wall thickness, mm & $11.1 \pm 1.1$ \\
End systolic volume, mL & $30.7 \pm 7.4$ \\
End diastolic volume, mL & $80.0 \pm 14.7$ \\
Stroke volume, $\mathrm{mL}$ & $49.2 \pm 9.5$ \\
Fractional shortening, FS\% & $37.0 \pm 6.3$ \\
Cardiac output, $\mathrm{mL} / \mathrm{min}$ & $3339.1 \pm 759.0$ \\
Left ventricular ejection fraction, LVEF\% & $61.6 \pm 5.6$ \\
\hline
\end{tabular}

Table 2. The Mean and Standard Deviation of the Basal Maximum Radius, the Apical Maximum Radius, the Distance Between Apical and Basal Levels (LV Length), Rotation Angles of Basal and Apical Levels and so LV Normalized Torsion Angle (Degree) in Short Axis View

\begin{tabular}{llllll}
\hline $\begin{array}{l}\text { Basal Radius, } \\
\mathbf{m m}\end{array}$ & $\begin{array}{l}\text { Apical Radius, } \\
\mathbf{m m}\end{array}$ & $\begin{array}{l}\text { LVLength, } \\
\mathbf{m m}\end{array}$ & $\begin{array}{l}\text { Basal Rotation Angle, } \\
\text { degree }\end{array}$ & $\begin{array}{l}\text { Apical Rotation Angle, } \\
\text { degree }\end{array}$ & $\begin{array}{l}\text { Normalized Torsion Angle, } \\
\text { degree }\end{array}$ \\
\hline & & & & & \\
$21.59 \pm 1.06$ & $17.73 \pm 2.08$ & $88.45 \pm 7.35$ & $7.96 \pm 1.57$ & $9.49 \pm 1.71$ & $7.80 \pm 1.27$ \\
\hline
\end{tabular}


pressure, left ventricular end diastolic/systolic volumes, interventricular septal diastole thickness, and posterior wall thickness measured in the long axis view of the left ventricle. Also, left ventricular ejection fraction (LVEF \%) was calculated using Simpson's biplane method by measuring end diastolic and end systolic volumes in 2D images. The global echocardiography parameter valuesstroke volume, fractional shortening, cardiac output, and left ventricular ejection fraction-suggested that

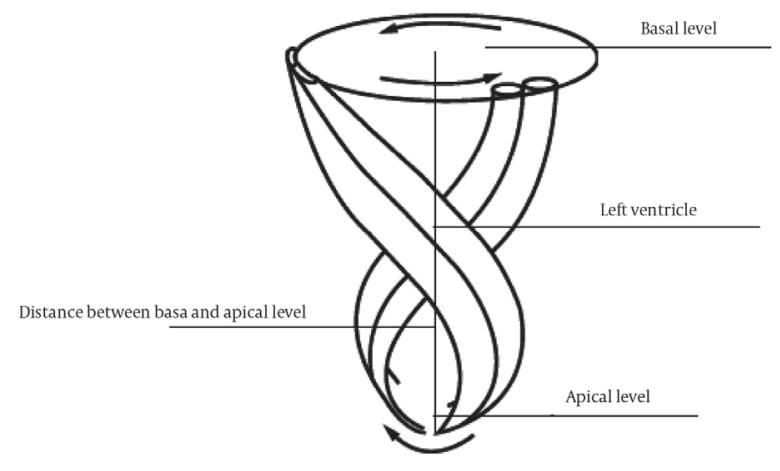

Figure 1. Rotiation Angle in Left Ventricular Chamber
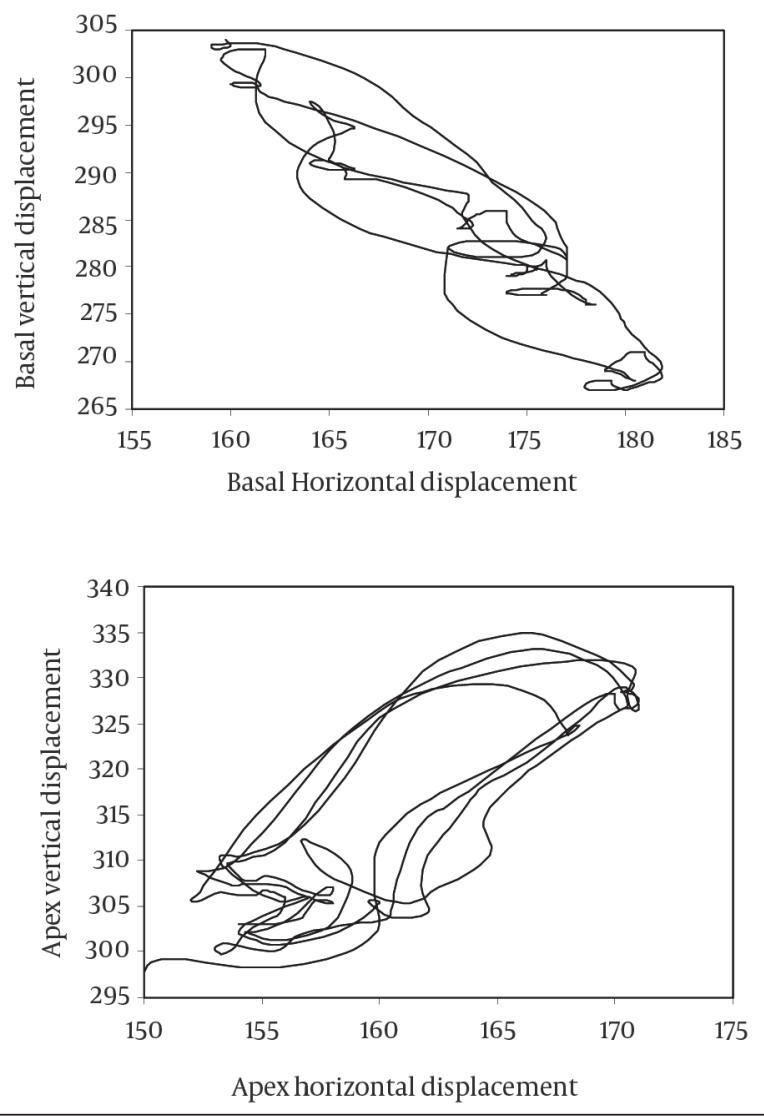

Figure 2. The Instantaneous Changes of the Vertical Displacement (Pixel) Relative to Horizontal Displacement (Pixel) of; a) Basal and, b) Apical Levels in Short Axis View Throughout Four Cardiac Cycles for one of the Subject; Extracted by Block Matching Algorithm.

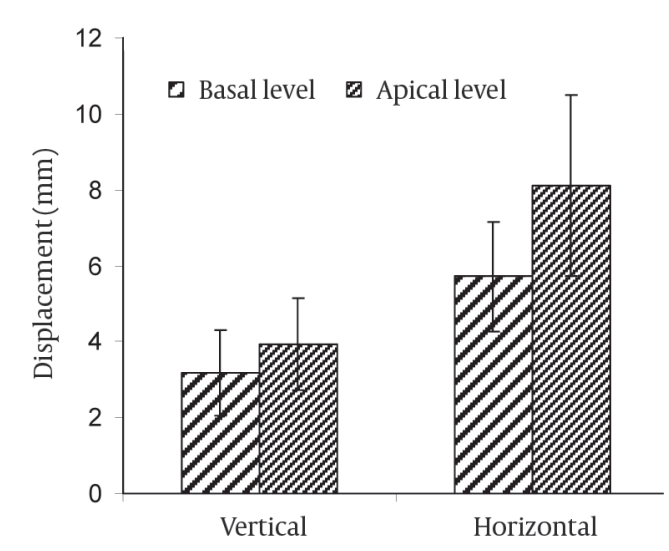

Figure 3. Mean and Standard Deviation of the Vertical and Horizontal Displacements (mm) of LV Myocardial Septal Wall in Basal and Apical Levels at Short Axis View for Healthy Subjects.

Figure 4. The LV Basal and Apical Rotation Angles and so Torsion Angle (Degree)

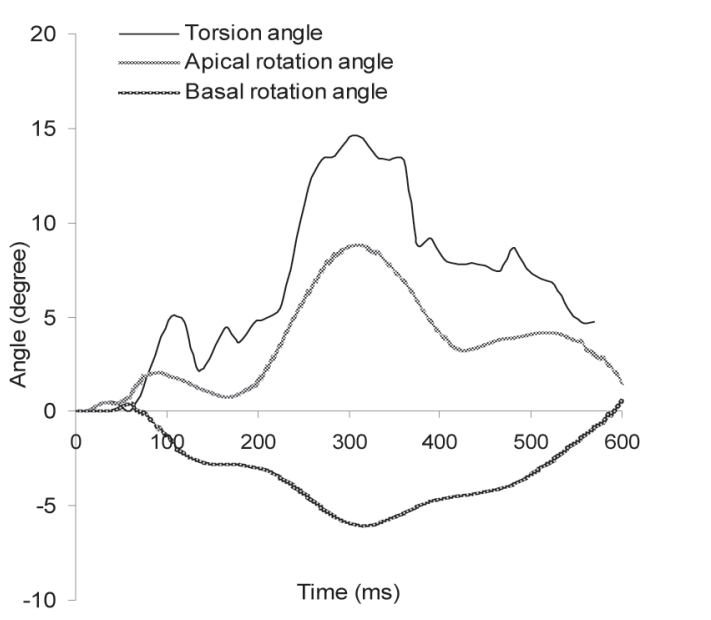

In short axis view during a cardiac cycle for one of the subject. The temporal resolution is $17 \mathrm{~ms}$.

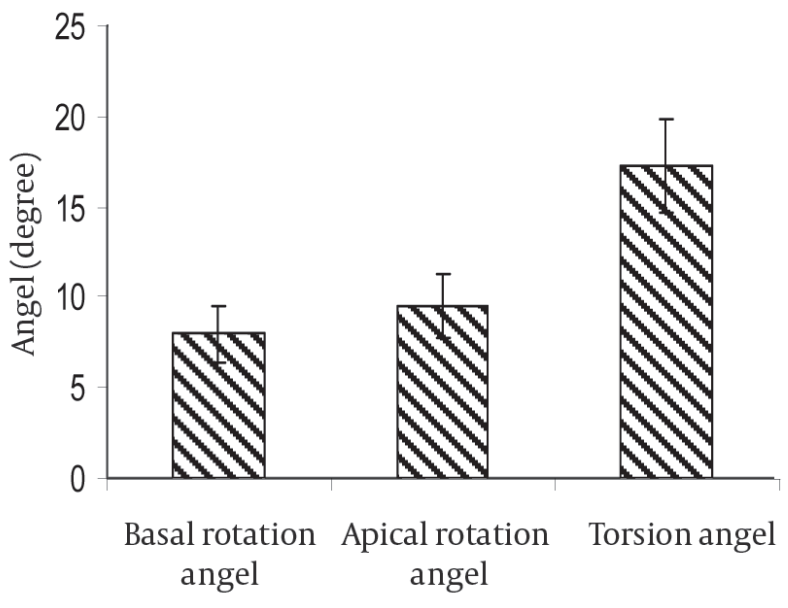

Figure 5. The Mean and Standard Deviation of Maximum Rotation Angles of Basal and Apical Levels and so LV Torsion Angle in Short Axis View 
subjects had healthy cardiac function without any heart disease symptoms.

For one of the subjects, the myocardium wall displacement waveforms at each frame using block matching are shown in Figure $2(a, b)$. In this figure, the instantaneous changes of the vertical displacement (pixel) relative to the horizontal displacement (pixel) of the basal and apical levels in the short axis view are presented throughout four cardiac cycles. Maximum displacement values of left ventricular myocardial septal wall in the basal and apical levels in the vertical and horizontal directions of the short axis view were estimated throughout four cardiac cycles by the BM algorithm for 14 healthy subjects. The mean and SD of the results are presented in Figure 3.

The results of the vertical and horizontal displacements of the apical level were more than the basal level than expected, because the LV apical level had more rotation relative to the basal level. All data are expressed as mean \pm standard deviation (SD). Data was tested for normal distribution and homogeneity of variance by the Kolmogorov-Smirnov test (K-S) and Levene's test respectively.

Extracting the instantaneous changes in total displacement vector in the short axis view throughout a cardiac cycle using the BM algorithm and, thus, the center of the desired levels in the end diastolic phase frame, we calculated the instantaneous changes in the rotation and torsion angles in the short axis view. For one of the subjects, the LV basal and apical rotation angles and torsion angle in the short axis view are shown in Figure 4.

The mean and standard deviation of the maximum rotation angles of the basal and apical levels and LV torsion angle in the short axis view are shown in Figure 5 for healthy subjects. To account for differences in heart size, normalized torsion was calculated based on the basal maximum radius, the apical maximum radius, and the distance between the apical and basal levels. The results are presented in Table 2. Torsion, as calculated in most echocardiographic studies, does not include adjustments for maximum apical and basal radii. Therefore, torsion measured by this method is directly related to the circumferential-longitudinal shear angle and appeared to have distorted geometries in pathological ventricles; this parameter may be important.

\section{Discussion}

Torsion angle is one of the biomechanical parameters that have attracted the attention of many researchers recently. The right-handle helix of the inner layer and the left-handle helix of the outer layer cause two torques on the two layers. Torque differences in systolic induce counterclockwise and clockwise rotations in the apical and basal systems, respectively (19). LV torsion meets its peak at the near end systolic and varies with changes in preload, afterload, and contractility; therefore, extraction of LV torsion is a cornerstone parameter of systolic function (6). Early strategies in measuring torsion were invasive, such as tracking the motion of metal markers implanted in animals and transplanted human hearts by biplane cine radiography $(20,21)$. However, more recent studies have reported noninvasive methods based on imaging modalities that can also quantify LV torsion with a high degree of accuracy.

Echocardiography is a safe and cost-efficient method of assessing cardiac anatomy and function. Twist angle was an early echo-based method, in which the motion of anatomical landmarks, such as the papillary muscles, was tracked over the cardiac cycle in a single short-axis slice $(22,23)$. Tissue Doppler imaging (TDI) is a unidimensional approach for calculating torsion from myocardial velocities at both the apex and base short-axis planes. Notomi et al. (24) showed that TDI can accurately measure LV rotational velocity with higher temporal resolution than MRI (25). As with any Doppler technique, the angle dependency of myocardial tissue velocity data is a limitation. Therefore, 2D speckle tracking imaging (STI) was proposed, which is independent of angle dependency factors (13). The speckle patterns within tissues are one of the characteristics of static B-mode echo imaging. These speckles are the result of constructive and destructive ultrasound interference backscatter from anatomical structures smaller than the wavelength of ultrasound. However, it is difficult to track the 2D motions of the myocardium using a speckle-tracking algorithm. The reported assessment of left ventricular torsion by speckle tracking depends on tissue motion from an isolated 2-dimensional plane, whereas myocardium has 3-dimensional motion; thus, some of the speckles might be lost from the view by intra-planar motion, particularly at the basal level. Then, the speckle-tracking algorithm can not trace the desired speckle pattern. But, using an echo tracking algorithm, it seems that we can trace motion with better accuracy. Therefore, in this study, we used echo tracking software that is able to use a block-matching algorithm for detecting echo patterns of images.

The alternative method for estimating motion proposed here is based on 2-dimensional echo tracking using time domain processing, an approach that should be independent of both cardiac translation and angle dependency. Success in the current study appears to derive from several factors, including the use of high-quality second-harmonic images that are acquired at high frame rates and are stored digitally in the original raw (scan line) format for analysis.

There are, however, several shortcomings to the current approach. The echo tracking algorithm analysis is inherently dependent on the 2-dimensional echo image quality. Although speckle tracking has been reported previously $(13,24)$, all of these methods have inferred tissue motion from isolated 2-dimensional planes, whereas this motion is actually 3-dimensional, which might remove some of the speckles from view by through-plane motion, particularly at the basal level. However, we tried to define each slice by anatomical landmarks of the LV, with characterized echo pattern. Therefore, efforts to combine the use of echo tracking technology with 3D 
echo are in the preliminary stages. Our study only investigated the changes in LV myocardial septal wall rotation and torsion in the short-axis view. If the images could be scanned with a better spatial resolution, it would be possible to study other segments of the myocardial wall. Because the frame rate in the images was not high enough, we could not evaluate clockwise rotation of the apex or counterclockwise rotation of the basal in the isovolumic contraction phase. In evaluating torsion angle in the short-axis view, we assumed that the short axis plane of the LV had a circular geometry, but because of a curved posterolateral wall and flat antrerior wall, the short axis plane does not have a circular shape (11). Also, torsion angle is a nonlinear function of ventricular length, and thus, angle magnitude depends on the place of reference levels; because choosing the reference levels in the same level in all subjects in the short axis view is difficult, torsion magnitude in the short-axis view has some variance between studies.

According to the statement above, we studied torsion angle on the short axis view. The vertical and horizontal displacements of the apical level are more than those of the basal level than expected, because the LV apical level has more rotation relative to the basal level. To account for differences in heart size, normalized torsion was calculated based on the maximum basal radius, the apical maximum radius, and the distance between the apical and basal levels. The normalized torsion angle and torsion angle for healthy subjects were $7.80 \pm 1.27$ and 17.26 \pm 2.53 degrees, respectively. The percentages of the coefficient of variation (CV\%) of the normalized torsion angle and torsion angle were $16 \%$ and $15 \%$, respectively.

Our study investigated the changes in LV myocardial septal wall rotation and the torsion in the short axis view, extracted with an echo pattern tracking algorithm. We conclude that echo pattern tracking is new method for motion detection in left ventricular to estimate torsion and rotation angles in the short axis view. It appears that the normalized torsion angle is a better biomechanical parameter than torsion angle, because it is independent of the heterogeneity in papillary muscle prominence and apical trabeculation in humans and distorted geometries in pathological ventricles.

\section{Acknowledgments}

This study was approved and funded by Tarbiat Modares University.

\section{Financial Disclosure}

There is no conflict of interests regarding the above paper.

\section{Funding/Support}

This work was supported by Tarbiat Modares University.

\section{References}

1. Bijnens BH, Cikes M, Claus P, Sutherland GR. Velocity and deformation imaging for the assessment of myocardial dysfunction.
Eur JEchocardiogr. 2009;10(2):216-26.

2. Helle-Valle T, Crosby J, Edvardsen T, Lyseggen E, Amundsen BH, Smith HJ, et al. New noninvasive method for assessment of left ventricular rotation: speckle tracking echocardiography. Circulation. 2005;112(20):3149-56.

3. Chan M. The world health report 2008: Primary health care.: World Health Organization (WHO); 2008; Available from: http:/ www.who.int/whr/2008/.

4. Bijnens B, Claus P, Weidemann F, Strotmann J, Sutherland GR Investigating cardiac function using motion and deformation analysis in the setting of coronary artery disease. Circulation. 2007;116(21):2453-64.

5. Atwood JD, Maltin L. Putting Eastern philosophies into Western psychotherapies. Am J Psychother. 1991;45(3):368-82.

6. Takeuchi M, Otsuji Y, Lang RM. Evaluation of left ventricular function using left ventricular twist and torsion parameters. Curr Cardiol Rep. 2009;11(3):225-30.

7. Yoon AJ, Bella JN. Echocardiographic assessment of left ventricular torsion. US Radiol. 2010;2:52-6.

8. Sengupta PP, Korinek J, Belohlavek M, Narula J, Vannan MA, Jahangir A, et al. Left ventricular structure and function: basic science for cardiac imaging. J Am Coll Cardiol. 2006;48(10):19882001.

9. Luo X, Cao T, Li Z, Duan Y. A preliminary study on the evaluation of relationship between left ventricular torsion and cardiac cycle phase by two-dimensional ultrasound speckle tracking imaging. Int J Cardiovasc Imaging. 2009;25(6):559-68.

10. Garot J, Pascal O, Diebold B, Derumeaux G, Gerber BL, DuboisRande JL, et al. Alterations of systolic left ventricular twist after acute myocardial infarction. Am J Physiol Heart Circ Physiol. 2002;282(1):H357-62.

11. Marwick TH, Yu CM, Sun JP. Technical principles of tissue velocity and strain imaging methods. In: Heimdal A, editor. Myocardial imaging tissue Doppler and speckle tracking. 1st ed. Massachusette: Wiley-Blackwell; 2007.

12. Behar V, Adam D, Lysyansky P, Friedman Z. Improving motion estimation by accounting for local image distortion. Ultrasonics. 2004;43(1):57-65.

13. Notomi Y, Lysyansky P, Setser RM, Shiota T, Popovic ZB, MartinMiklovic MG, et al. Measurement of ventricular torsion by twodimensional ultrasound speckle tracking imaging. J Am Coll Cardiol. 2005;45(12):2034-41.

14. Grundy SM, Pasternak R, Greenland P, Smith S, Jr., Fuster V. Assessment of cardiovascular risk by use of multiple-risk-factor assessment equations: a statement for healthcare professionals from the American Heart Association and the American College of Cardiology. Circulation. 1999;100(13):1481-92.

15. Anderson KM, Wilson PW, Odell PM, Kannel WB. An updated coronary risk profile. A statement for health professionals. Circulation. 1991;83(1):356-62.

16. Henry WL, DeMaria A, Gramiak R, King DL, Kisslo JA, Popp RL, et al. Report of the American Society of Echocardiography Committee on Nomenclature and Standards in Two-dimensional Echocardiography. Circulation. 1980;62(2):212-7.

17. Yeung F, Levinson SF, Fu D, Parker KJ. Feature-adaptive motion tracking of ultrasound image sequences using a deformable mesh. IEEE Trans Med Imaging. 1998;17(6):945-56.

18. Soleimani E, Mokhtari Dizaji M, Saberi H. Carotid artery wall motion estimation from consecutive ultrasonic images: Comparison between block-matching and maximum-gradient algorithms. $J$ Teh Univ Heart Ctr. 2011;6(2):72-8.

19. Hill HZ, Hill GJ. Irradiation of cells attached or suspended by rubber policeman or by trypsin influences the extent of DNA strand breaks induced by ionizing radiation. Radiat Res. 1991;125(3):343-5.

20. Ingels NB, Jr., Hansen DE, Daughters GT, 2nd, Stinson EB, Alderman EL, Miller DC. Relation between longitudinal, circumferential, and oblique shortening and torsional deformation in the left ventricle of the transplanted human heart. Circ Res. 1989;64(5):915-27.

21. Hansen DE, Daughters GT, 2nd, Alderman EL, Stinson EB, Baldwin JC, Miller DC. Effect of acute human cardiac allograft rejection on left ventricular systolic torsion and diastolic recoil measured by intramyocardial markers. Circulation. 1987;76(5):998-1008.

22. Mirro MJ, Rogers EW, Weyman AE, Feigenbaum H. Angular dis- 
placement of the papillary muscles during the cardiac cycle. Circulation. 1979;60(2):327-33.

23. Arts T, Meerbaum S, Reneman RS, Corday E. Torsion of the left ventricle during the ejection phase in the intact dog. Cardiovasc Res. 1984;18(3):183-93.

24. Notomi Y, Setser RM, Shiota T, Martin-Miklovic MG, Weaver JA,
Popovic ZB, et al. Assessment of left ventricular torsional deformation by Doppler tissue imaging: validation study with tagged magnetic resonance imaging. Circulation. 2005;111(9):1141-7.

25. Kim HW, Crowley AL, Kim RJ. A clinical cardiovascular magnetic resonance service: operational considerations and the basic examination. Magn Reson Imaging Clin N Am. 2007;15(4):473-85, v. 